\title{
Adaptive NN State-Feedback Control for Stochastic High-Order Nonlinear Systems with Time-Varying Control Direction and Delays
}

\author{
Huifang Min and Na Duan \\ School of Electrical Engineering \& Automation, Jiangsu Normal University, Xuzhou 221116, China \\ Correspondence should be addressed to Na Duan; duanna08@163.com
}

Received 11 March 2015; Accepted 14 May 2015

Academic Editor: Asier Ibeas

Copyright (C) 2015 H. Min and N. Duan. This is an open access article distributed under the Creative Commons Attribution License, which permits unrestricted use, distribution, and reproduction in any medium, provided the original work is properly cited.

Nussbaum-type gain function and neural network $(\mathrm{NN})$ approximation approaches are extended to investigate the adaptive statefeedback stabilization problem for a class of stochastic high-order nonlinear time-delay systems. The distinct features of this paper are listed as follows. Firstly, the power order condition is completely removed; the restrictions on system nonlinearities and time-varying control direction are greatly weakened. Then, based on Lyapunov-Krasovskii function and dynamic surface control technique, an adaptive NN controller is constructed to render the closed-loop system semiglobally uniformly ultimately bounded (SGUUB). Finally, a simulation example is shown to demonstrate the effectiveness of the proposed control scheme.

\section{Introduction}

During the past decades, the control of stochastic nonlinear systems has been an interesting field and received fruitful development based on the stochastic stability theory in [1-3] and other references. However, the existences of nonlinearities and time delays cause the instability of system performance and give rise to much greater design difficulty in the stabilization procedure. To handle nonlinearities, neural network $(\mathrm{NN})$, especially radial basis function neural network (RBF NN), has been successfully extended to stochastic nonlinear systems due to its inherent approximation capacity; see $[4-8]$ and the references therein. To deal with time delays, there are often two ways, namely, Lyapunov-Krasovskii function and Lyapunov-Razumikhin technique. Recently, together with NN and backstepping, Lyapunov-Krasovskii theory was used in [9-13] and Lyapunov-Razumikhin approach was utilized in [14-17] to stabilize stochastic nonlinear systems with time delays, respectively. However, to the best of our knowledge, for stochastic high-order nonlinear time-delay systems, there are still a few results.
In this paper, we further consider the following stochastic high-order time-delay system:

$$
\begin{aligned}
d x_{i}= & x_{i+1}^{p_{i}} d t+f_{i}\left(t, x(t), x\left(t-d_{i}(t)\right)\right) d t \\
& +g_{i}\left(t, x(t), x\left(t-d_{i}(t)\right)\right) d \omega, \\
\quad i=1, \ldots, n-1, & \\
d x_{n}= & \eta(t) u^{p_{n}} d t+f_{n}\left(t, x(t), x\left(t-d_{n}(t)\right)\right) d t \\
& +g_{n}\left(t, x(t), x\left(t-d_{n}(t)\right)\right) d \omega, \\
y= & x_{1},
\end{aligned}
$$

where $x=\left(x_{1}, \ldots, x_{n}\right)^{T} \in \mathbb{R}^{n}$ and $x\left(t-d_{i}(t)\right)=\left(x_{1}(t-\right.$ $\left.\left.d_{i}(t)\right), \ldots, x_{n}\left(t-d_{i}(t)\right)\right)^{T}$ are system state vectors; $x_{1}, \ldots, x_{n}$, $u \in \mathbb{R}$ and $y$ are system measurable states, control input and output, respectively. For $1 \leq i \leq n, d_{i}(t): \mathbb{R}^{+} \rightarrow\left[0, d_{i}\right]$ are time-varying delays, which are Borel measurable functions. Define $d=\max _{1 \leq i \leq n}\left\{d_{i}\right\}$ with initial value $\{x(\theta):-d \leq$ $\theta \leq 0\}=\xi \in \mathscr{C}_{\mathscr{F}_{0}}^{b}\left([-d, 0] ; \mathbb{R}^{n}\right) \cdot p_{1}, \ldots, p_{n} \geq 1$ are odd 
integers called the high-order of the system. $\eta(t) \neq 0$ is unknown bounded time-varying continuous function called control direction. For $1 \leq i \leq n$, the drift terms $f_{i}: \mathbb{R}^{+} \times$ $\mathbb{R}^{n} \times \mathbb{R}^{n} \rightarrow \mathbb{R}$ and the diffusion terms $g_{i}: \mathbb{R}^{+} \times \mathbb{R}^{n} \times$ $\mathbb{R}^{n} \rightarrow \mathbb{R}^{r}$ are assumed to be locally Lipschitz in $(x(t), x(t-$ $\left.\left.d_{i}(t)\right)\right)$ and piecewise continuous in $t$ with $f_{i}(t, 0,0)=0$ and $g_{i}(t, 0,0)=0 . \omega$ is an $r$-dimensional standard Wiener process defined on a probability space $\left\{\Omega, \mathscr{F},\{\mathscr{F}\}_{t>0}, P\right\}$, where $\Omega$ is a sample space, $\mathscr{F}$ is a $\sigma$-field, $\{\mathscr{F}\}_{t \geq 0}$ is filtration, and $P$ is the probability measure.

When $d_{i}(t)=0,[18-27]$ investigated different feedback control problems for system (1) with different structures. When $d_{i}(t) \neq 0$, [28] studied the output-feedback control with $p_{i}=p$ and $d_{i}(t)=d(t)$ under restrictive growth conditions on $f_{i}$ and $g_{i}$. To relax the growth conditions, $[29,30]$ firstly generalized the homogeneous domination approach proposed by [31] to stochastic high-order time-delay systems. Subsequently, [32-34] further solved the stabilization problems for more general systems based on homogeneous domination approach. However, in [29, 30, 32-34], the nonlinear functions were still assumed to satisfy somewhat restrictive growth conditions. In addition, [29, 30, 32-34] were all achieved with $\eta(t)$ being bounded by positive constants or $\eta(t)=1$. For $\eta(t) \neq 1$, the Nussbaum-type gain function approach proposed by [35] has been proven to be a useful tool and is frequently used in [11, 12, 36-38] for stochastic nonlinear systems with $p_{i}=1$. Immediately, one raises the following problem:

How does one generalize RBF NN and Nussbaum-type gain function approaches to system (1) to further relax the restrictions on nonlinear functions $f_{i}$ and $g_{i}$ and time-varying control direction $\eta(t)$ ?

This paper focuses on solving this problem. The main contributions are listed as follows. (i) Compared with the existing results $[29,30,32-34]$, we largely weaken the restrictions on the drift and diffusion terms by extending NN approximation approach to system (1). Furthermore, to handle unknown control direction, we utilize Nussbaumtype gain function approach to allow the sign and the bounds of $\eta(t)$ to be unknown. (ii) By introducing dynamic surface control (DSC), the problem of "explosion of complexity" generalized by the repeated differentiation of virtual controllers is avoided, which greatly simplifies the control algorithm. (iii) An adaptive state-feedback controller is constructed to ensure the closed-loop system to be semiglobally uniformly ultimately bounded (SGUUB) by using backstepping technique and choosing Lyapunov-Krasovskii function skillfully.

The remainder of this paper is organized as follows. Section 2 begins with mathematical preliminaries. The design process and analysis of adaptive state-feedback controller are given in Sections 3 and 4, respectively. In Section 5, a simulation example is shown. Section 6 summarizes the paper. Finally, the necessary proof is provided in the Appendix.

\section{Mathematical Preliminaries}

The following notations, definitions, and lemmas are to be used.

Notations. $\mathbb{R}^{+}$denotes the set of all the nonnegative real numbers; $\mathbb{R}^{n}$ denotes the $n$-dimensional Euclidean space. $\mathscr{C}^{i}$ denotes the family of all the functions with continuous $i$ th partial derivations; $\mathscr{C}^{2,1}\left(\mathbb{R}^{n} \times[-d, \infty), \mathbb{R}^{+}\right)$denotes the family of all nonnegative functions $V(x, t)$ on $\mathbb{R}^{n} \times[-d, \infty)$ which are $\mathscr{C}^{2}$ in $x$ and $\mathscr{C}^{1}$ in $t . \mathscr{C}\left([-d, 0] ; \mathbb{R}^{n}\right)$ denotes the space of continuous $\mathbb{R}^{n}$-valued functions on $[-d, 0]$ endowed with the norm $\|\cdot\|$ defined by $\|f\|=\sup _{x \in[-d, 0]}|f(x)|$ for $f \in$ $\mathscr{C}\left([-d, 0], \mathbb{R}^{n}\right) ;|\cdot|$ denotes the Euclidean norm of a vector; $\mathscr{C}_{\mathscr{F}_{0}}^{b}\left([-d, 0], \mathbb{R}^{n}\right)$ denotes the family of all $\mathscr{F}_{0}$-measurable bounded $\mathscr{C}\left([-d, 0] ; \mathbb{R}^{n}\right)$-valued random variables $\xi=\{\xi(\theta)$ : $-d \leq \theta \leq 0\} . X^{T}$ denotes the transpose of a given vector or matrix $X ; \operatorname{Tr}\{X\}$ denotes its trace when $X$ is square. $\mathscr{K}$ denotes the set of all functions $\mathbb{R}^{+} \rightarrow \mathbb{R}^{+}$, which are continuous, strictly increasing, and vanishing at zero; $\mathscr{K}_{\infty}$ denotes the set of all functions which are of class $\mathscr{K}$ and unbounded. To simplify the procedure, we sometimes denote $X(t)$ by $X$ for any variable $X(t)$.

Consider the following stochastic time-delay system:

$$
\begin{aligned}
d x(t)= & f(t, x(t), x(t-d(t))) d t \\
& +g(t, x(t), x(t-d(t))) d \omega, \quad \forall t \geq 0,
\end{aligned}
$$

with initial data $\{x(\theta):-d \leq \theta \leq 0\}=\xi \in \mathscr{C}_{\mathscr{F}_{0}}^{b}\left([-d, 0], \mathbb{R}^{n}\right)$, where $d(t): \mathbb{R}^{+} \rightarrow[0, d]$ is a Borel measurable function; $\omega$ is an $r$-dimensional standard wiener process defined as in (1). $f: \mathbb{R}^{+} \times \mathbb{R}^{n} \times \mathbb{R}^{n} \rightarrow \mathbb{R}^{n}$ and $g: \mathbb{R}^{+} \times \mathbb{R}^{n} \times \mathbb{R}^{n} \rightarrow \mathbb{R}^{n \times r}$ are locally Lipschitz with $f(0,0, t)=0$ and $g(0,0, t)=0$. For any given $V(x(t), t) \in C^{2,1}$, together with stochastic system (2), the differential operator $\mathscr{L}$ is defined as

$$
\mathscr{L} V=\frac{\partial V}{\partial t}+\frac{\partial V}{\partial x} f+\frac{1}{2} \operatorname{Tr}\left\{g^{T} \frac{\partial^{2} V}{\partial x^{2}} g\right\} .
$$

Definition 1 (see [35]). A function $N(\zeta)$ is called a Nussbaumtype function if it has the following properties:

$$
\begin{gathered}
\limsup _{s \rightarrow \infty} \int_{s_{0}}^{s} N(\zeta) d \zeta=+\infty, \\
\liminf _{s \rightarrow \infty} \int_{s_{0}}^{s} N(\zeta) d \zeta=-\infty .
\end{gathered}
$$

Many functions satisfy these properties, for example, $e^{\zeta^{2}} \cos (\pi \zeta / 2), \ln (\zeta+1) \cos (\sqrt{\ln (\zeta+1)})$, and $\zeta^{2} \cos (\pi \zeta / 2)$. Throughout this paper, the Nussbaum function $N(\zeta)=$ $e^{\zeta^{2}} \cos (\pi \zeta / 2)$ is exploited.

Definition 2 (see [14]). Let $p \geq 1$; considering stochastic nonlinear time-delay system (2), the solution $\{x(t), t \geq 0\}$ with initial condition $\xi \in S_{0}$ ( $S_{0}$ is some compact set containing the origin) is said to be $p$-moment semiglobally uniformly ultimately bounded (SGUUB) if there exists a constant $d$ such that $E\left\{\|x(t, \xi)\|^{p}\right\} \leq d, \forall t \geq T$, holds for some $T \geq 0$. 
To facilitate the control design, the following lemmas are applied.

Lemma 3 (Young's inequality). For $\forall(x, y) \in \mathbb{R}^{2}, x y \leq$ $\left(\varepsilon^{p} / p\right)|x|^{p}+\left(1 / q \varepsilon^{q}\right)|y|^{q}$ holds, where $\varepsilon>0, p, q>1$, and $1 / p+1 / q=1$.

Lemma 4 (see [26]). For real variables $x \geq 0$ and $y>0$; then, $x \leq y+(x / m)^{m}((m-1) / y)^{m-1}$, where $m \geq 1$ is a real number.

Lemma 5 (see [31]). Let $x$ and $y$ be real variables; then, for any real numbers $m, n, b>0$ and continuous function $a(\cdot) \geq 0$, one has $a(\cdot) x^{m} y^{n} \leq b|x|^{m+n}+(n /(m+n))((m+$ $n) / m)^{-m / n} a(\cdot)^{(m+n) / n} b^{-m / n}|y|^{m+n}$.

Lemma 6 (see [31]). For $x, y \in \mathbb{R}$, where $p \geq 1$ is a constant, the following inequalities hold: $|x+y|^{p} \leq 2^{p-1}\left|x^{p}+y^{p}\right|$ and $(|x|+|y|)^{1 / p} \leq|x|^{1 / p}+|y|^{1 / p}$.

In the sequel, radial basis function neural network (RBF $\mathrm{NN}$ ) is to be applied to estimate the unknown nonlinear functions. By choosing sufficiently large node number, for any continuous function $f(x)$ over a compact set $S_{x} \subset \mathbb{R}^{q}$, there is a RBF NN $W^{*^{T}} S(x)$ such that, for an ideal level of accuracy $\varepsilon(0<\varepsilon<1)$,

$$
f(x)=W^{*^{T}} S(x)+\delta(x), \quad|\delta(x)| \leq \varepsilon,
$$

where $\delta(x)$ is the approximation error and $S(x)=\left[s_{1}(x), \ldots\right.$, $\left.s_{N}(x)\right]^{T}$ is the known function vector with $N>1$ being the RBF NN node number. The basis functions $s_{i}(x)(1 \leq i \leq N)$ are chosen as $s_{i}(x)=\exp \left[-\left(x-b_{i}\right)^{T}\left(x-b_{i}\right) / \varsigma^{2}\right]$, where $\varsigma$ is the width of the function and $b_{i}=\left[b_{i 1}, \ldots, b_{i n}\right]^{T}$ is the center of the receptive field. $W^{*}$ is the ideal constant weight vector with the form $W^{*}=\arg \min _{W \in \mathbb{R}^{N}}\left\{\sup _{x \in S_{x}}\left|f(x)-W^{T} S(x)\right|\right\}$, where arg min is the value of variable $W$ when the objective function $\sup _{x \in S_{x}}\left|f(x)-W^{T} S(x)\right|$ is minimum with $W=$ $\left[w_{1}, \ldots, w_{N}\right]^{T}$ being the weight vector.

\section{Design of State-Feedback Controller}

The objective of this paper is to design an adaptive NN statefeedback controller for system (1) under weaker conditions such that the closed-loop system is SGUUB. To achieve the above objective, we need the following assumptions.

Assumption 7. The time-varying delays $d_{i}(t), i=1, \ldots, n$, in system (1) satisfy $0 \leq d_{i}(t) \leq h_{i}$ and $\dot{d}_{i}(t) \leq \gamma_{i}<1$ for positive constants $h_{i}$ and $\gamma_{i}$.

Assumption 8. $\eta(t) \neq 0$ is unknown sign and takes value in the unknown closed interval $H:=\left[l^{-}, l^{+}\right]$with $0 \notin H$.

Assumption 9. Nonlinear functions $f_{i}$ and $g_{i}$ satisfy

$$
\begin{aligned}
& \left|f_{i}\left(t, x(t), x\left(t-d_{i}(t)\right)\right)\right| \\
& \quad \leq f_{i 1}(y)+f_{i 2}\left(y\left(t-d_{i}(t)\right)\right),
\end{aligned}
$$

$$
\begin{aligned}
& \left|g_{i}\left(t, x(t), x\left(t-d_{i}(t)\right)\right)\right| \\
& \quad \leq g_{i 1}(y)+g_{i 2}\left(y\left(t-d_{i}(t)\right)\right),
\end{aligned}
$$

for $i=1, \ldots, n$, where $f_{i 1}, f_{i 2}, g_{i 1}$, and $g_{i 2}$ are positive functions with $f_{i j}(s)=s \bar{f}_{i j}(s)$ and $g_{i j}(s)=s \bar{g}_{i j}(s)$ for $j=1,2$.

Remark 10. We emphasize two points. (i) To the best of our knowledge, only [33] consider the unknown control directions for stochastic high-order time-delay systems. However, in [33], the unknown control directions are of known signs and are bounded by positive constants. We allow the sign of $\eta(t)$ to be unknown and remove the bounds limitations in Assumption 8. (ii) Motivated by $[14,15]$ for stochastic time-delay systems, the restrictions on $f_{i}$ and $g_{i}$ are greatly relaxed in Assumption 9 compared with the existing results in $[29,30,32-34]$.

To simplify the design process, define

$$
\theta=\max \left\{N_{i}\left|W_{i}^{*}\right|^{2}, i=1, \ldots, n\right\},
$$

where $N_{1}, \ldots, N_{n}$ are the number of RBF NN nodes and $W_{1}^{*}, \ldots, W_{n}^{*}$ are the ideal constant weight vectors. Before the design procedure, introduce the following coordinate transformation:

$$
\begin{aligned}
z_{1} & =x_{1}, \\
z_{i} & =x_{i}-\alpha_{i f}, \quad i=2, \ldots, n,
\end{aligned}
$$

where $\alpha_{2 f}, \ldots, \alpha_{n f}$ are the outputs of the first-order filter with virtual control laws $\alpha_{2}, \ldots, \alpha_{n}$ being inputs. Now, we give the backstepping design procedure by utilizing the technique of DSC and RBF NN approximation approach.

Step 1. Choosing the 1st Lyapunov function candidate as $V_{1}\left(z_{1}, \widehat{\theta}\right)=\left(k_{1} /\left(p-p_{1}+4\right)\right) z_{1}^{p-p_{1}+4}+(1 / 2 \Gamma) \widetilde{\theta}$ and using (1), (3), and (8), one has

$$
\begin{aligned}
\mathscr{L} V_{1} \leq & k_{1} z_{1}^{p-p_{1}+3}\left(x_{2}^{p_{1}}+f_{1}(\cdot)\right) \\
& +\frac{p-p_{1}+3}{2} k_{1} z_{1}^{p-p_{1}+2} g_{1}(\cdot) g_{1}^{T}(\cdot)-\frac{1}{\Gamma} \widetilde{\theta} \dot{\hat{\theta}},
\end{aligned}
$$

where $p=\max \left\{p_{i}, i=1, \ldots, n\right\}, k_{1}, \Gamma>0$ are design constants, $\widehat{\theta}$ is the estimate of $\theta$, and $\widetilde{\theta}=\theta-\widehat{\theta}$ is the estimation error of $\theta$.

In the sequel, we estimate the terms of (9) by using Lemmas 4-5 and Assumption 9 as

$$
\begin{aligned}
& k_{1} z_{1}^{p-p_{1}+3} f_{1}(\cdot) \\
& \leq k_{1} z_{1}^{p-p_{1}+3}\left(f_{11}(y)+f_{12}\left(y\left(t-d_{1}(t)\right)\right)\right) \\
& \leq k_{1} z_{1}^{p-p_{1}+4} \bar{f}_{11}(y)+l_{11} z_{1}^{p-p_{1}+4} \\
& \quad+\bar{l}_{11} f_{12}^{p-p_{1}+4}\left(y\left(t-d_{1}(t)\right)\right) \leq k_{1} z_{1}^{p-p_{1}+4} \bar{f}_{11}(y) \\
& +l_{11} z_{1}^{p-p_{1}+4}+\xi_{11}+\bar{\xi}_{11} f_{12}^{p+3}\left(y\left(t-d_{1}(t)\right)\right),
\end{aligned}
$$




$$
\begin{aligned}
& \frac{p-p_{1}+3}{2} k_{1} z_{1}^{p-p_{1}+2} g_{1}(\cdot) g_{1}^{T}(\cdot) \leq k_{1}\left(p-p_{1}+3\right) \\
& \cdot z_{1}^{p-p_{1}+2}\left(g_{11}^{2}(y)+g_{12}^{2}\left(y\left(t-d_{1}(t)\right)\right)\right) \\
& \quad \leq k_{1}\left(p-p_{1}+3\right) z_{1}^{p-p_{1}+4} \bar{g}_{11}^{2}(y)+l_{12} z_{1}^{p-p_{1}+4} \\
& \quad+\bar{l}_{12} g_{12}^{p-p_{1}+4}\left(y\left(t-d_{1}(t)\right)\right) \leq k_{1}\left(p-p_{1}+3\right) \\
& \cdot z_{1}^{p-p_{1}+4} \bar{g}_{11}^{2}(y)+l_{12} z_{1}^{p-p_{1}+4}+\xi_{12} \\
& +\bar{\xi}_{12} g_{12}^{p+3}\left(y\left(t-d_{1}(t)\right)\right)
\end{aligned}
$$

where $l_{11}, l_{12}, \xi_{11}$, and $\xi_{12}$ are positive parameters, $\bar{l}_{11}=$ $\left(1 /\left(p-p_{1}+4\right)\right)\left(\left(\left(p-p_{1}+4\right) /\left(p-p_{1}+3\right)\right) l_{11}\right)^{-\left(p-p_{1}+3\right)} k_{1}^{p-p_{1}+4}$, $\bar{\xi}_{11}=\left(\left(\left(p-p_{1}+4\right) /(p+3)\right) \bar{l}_{11}\right)^{(p+3) /\left(p-p_{1}+4\right)}\left(\left(p_{1}-1\right) /(p-\right.$ $\left.\left.p_{1}+4\right) \xi_{11}\right)^{\left(p_{1}-1\right) /\left(p-p_{1}+4\right)}, \bar{l}_{12}=\left(2 /\left(p-p_{1}+4\right)\right)\left(\left(\left(p-p_{1}+\right.\right.\right.$ $\left.\left.4) /\left(p-p_{1}+2\right)\right) l_{12}\right)^{-\left(p-p_{1}+2\right) / 2}\left(k_{1}\left(p-p_{1}+3\right)\right)^{\left(p-p_{1}+4\right) / 2}$, and $\bar{\xi}_{12}=\left(\left(\left(p-p_{1}+4\right) /(p+3)\right) \bar{l}_{12}\right)^{(p+3) /\left(p-p_{1}+4\right)}\left(\left(p_{1}-1\right) /(p-\right.$ $\left.\left.p_{1}+4\right) \xi_{12}\right)^{\left(p_{1}-1\right) /\left(p-p_{1}+4\right)}$ are constants that can be designed. Substituting (10) into (9) and choosing the intermediate variable $\widehat{\alpha}_{2 f}$ as

$$
\widehat{\alpha}_{2 f}^{p_{1}}\left(X_{1}\right)=-\frac{z_{1}\left(k_{1} \bar{f}_{11}+k_{1}\left(p-p_{1}+3\right) \bar{g}_{11}^{2}+l_{11}+l_{12}\right)+\left(v_{1}(\cdot)+\phi_{12}(\widehat{\theta})\right) z_{1}^{p_{1}}}{k_{1}},
$$

one yields

$$
\begin{aligned}
& \mathscr{L} V_{1} \leq k_{1} z_{1}^{p-p_{1}+3}\left(x_{2}^{p_{1}}-\widehat{\alpha}_{2 f}^{p_{1}}\right)-v_{1}(\cdot) z_{1}^{p+3} \\
& -\phi_{12}(\widehat{\theta}) z_{1}^{p+3}-\frac{1}{\Gamma} \tilde{\theta} \dot{\hat{\theta}}+\xi_{11}+\xi_{12} \\
& +y^{p+3}\left(t-d_{1}(t)\right) S_{1}\left(y\left(t-d_{1}(t)\right)\right),
\end{aligned}
$$

where $X_{1}=\left(z_{1}, \widehat{\theta}\right) \in S_{X_{1}}=\left\{X_{1} \mid x \in S_{x}\right\}, S_{x}$ is a compact set through which the state trajectories may travel, $v_{1}(\cdot)$ and $\phi_{12}(\hat{\theta})$ are continuous functions to be designed later, and $S_{1}\left(y\left(t-d_{1}(t)\right)\right)=\bar{\xi}_{11} \bar{f}_{12}^{p+3}\left(y\left(t-d_{1}(t)\right)\right)+\bar{\xi}_{12} \bar{g}_{12}^{p+3}\left(y\left(t-d_{1}(t)\right)\right)$.

To proceed further, applying Lemmas 3-4 and RBF NN (5) to estimate $\widehat{\alpha}_{2 f}^{p_{1}}$ as $\widehat{\alpha}_{2 f}^{p_{1}}=W_{1}^{*^{T}} S_{1}+\delta_{1}$ and $\left|\delta_{1}\right|<\varepsilon_{1}$, this leads to

$$
\begin{aligned}
-k_{1} z_{1}^{p-p_{1}+3} \widehat{\alpha}_{2 f}^{p_{1}} & \leq z_{1}^{p-p_{1}+3}\left(k_{1}^{2}+\frac{\theta}{2}+\frac{\varepsilon_{1}^{2}}{2}\right) \\
& \leq z_{1}^{p-p_{1}+3} \varphi_{11}(\widehat{\theta})+\frac{1}{2} z_{1}^{p-p_{1}+3} \widetilde{\theta} \\
& \leq \xi_{1}+z_{1}^{p+3} \phi_{11}(\widehat{\theta})+\frac{1}{2} z_{1}^{p-p_{1}+3} \widetilde{\theta}
\end{aligned}
$$

where $\xi_{1}>0$ is a design constant, $\left|W_{1}^{*^{T}}\right|^{2} S_{1}^{2} \leq\left|W_{1}^{*^{T}}\right|^{2} N_{1} \leq \theta$, $\varphi_{11}(\widehat{\theta})=k_{1}^{2}+\sqrt{1+\widehat{\theta}^{2}} / 2+\varepsilon_{1}^{2} / 2$ and $\phi_{11} \geq\left(\left(\left(p-p_{1}+3\right) /(p+\right.\right.$ 3)) $\left.\varphi_{11}(\widehat{\theta})\right)^{(p+3) /\left(p-p_{1}+3\right)}\left(p_{1} /\left(p-p_{1}+3\right) \xi_{1}\right)^{p_{1} /\left(p-p_{1}+3\right)}$. Choose the first control law as

$$
\alpha_{2}\left(z_{1}, \widehat{\theta}\right)=-z_{1}\left(\frac{c_{1}+\phi_{11}(\widehat{\theta})}{k_{1}}\right)^{1 / p_{1}}=-z_{1} \beta_{1}(\widehat{\theta})
$$

which together with (13) changes (12) into

$$
\begin{aligned}
\mathscr{L} V_{1} \leq & -c_{1} z_{1}^{p+3}+k_{1} z_{1}^{p-p_{1}+3}\left(x_{2}^{p_{1}}-\alpha_{2}^{p_{1}}\right)-v_{1}(\cdot) z_{1}^{p+3} \\
& -\phi_{12}(\widehat{\theta}) z_{1}^{p+3}-\frac{1}{\Gamma} \widetilde{\theta}\left(\dot{\hat{\theta}}-\tau_{1}\right)+\Delta_{1} \\
& +y^{p+3}\left(t-d_{1}(t)\right) S_{1}\left(y\left(t-d_{1}(t)\right)\right)
\end{aligned}
$$

where $c_{1}>0, \tau_{1}=(\Gamma / 2) z_{1}^{p-p_{1}+3}$, and $\Delta_{1}=\xi_{1}+\xi_{11}+\xi_{12}$.

In order to avoid the repeated differentiation of virtual control law, we introduce dynamic surface control technique. Let $\alpha_{2 f}$ be obtained by a first-order filter with time constant $q_{2}$; then, it has $q_{2} \dot{\alpha}_{2 f}+\alpha_{2 f}=\alpha_{2}$ and $\alpha_{2 f}(0)=\alpha_{2}(0)$. Define $Y_{2}=\alpha_{2 f}-\alpha_{2}$ as the output error of the filter; thus, $\dot{\alpha}_{2 f}=-Y_{2} / q_{2}$ and $\dot{Y}_{2}=-Y_{2} / q_{2}+D_{2}\left(X_{1}\right)$, where $D_{2}\left(X_{1}\right)=$ $\dot{z}_{1} \beta_{1}(\widehat{\theta})+z_{1} \dot{\beta}_{1}(\widehat{\theta}) \dot{\hat{\theta}}$. Then, by using Lemmas 5-6 and $(a+b)^{n}=$ $\sum_{i=0}^{n} C_{n}^{i} a^{n-i} b^{i}$, it holds that

$$
\begin{aligned}
& k_{1} z_{1}^{p-p_{1}+3}\left(x_{2}^{p_{1}}-\alpha_{2}^{p_{1}}\right)=k_{1} z_{1}^{p-p_{1}+3}\left(\left(z_{2}+Y_{2}+\alpha_{2}\right)^{p_{1}}\right. \\
& \left.-\alpha_{2}^{p_{1}}\right) \leq k_{1} z_{1}^{p-p_{1}+3}\left(\sum_{j=0}^{p_{1}-1} C_{p_{1}}^{j}\left(z_{2}+Y_{2}\right)^{p_{1}-j}\right. \\
& \left.\cdot\left(-z_{1} \beta_{1}(\widehat{\theta})\right)^{j}\right) \leq k_{1} \sum_{j=0}^{\left(p_{1}-1\right) / 2} C_{p_{1}}^{2 j} 2^{p_{1}-1-2 j} z_{1}^{p-p_{1}+3+2 j} \\
& \cdot\left(\left|z_{2}\right|^{p_{1}-2 j}+\left|Y_{2}\right|^{p_{1}-2 j}\right) \beta_{1}^{2 j} \leq \gamma_{11} z_{1}^{p+3}+\phi_{20}(\widehat{\theta}) \\
& \cdot z_{2}^{p+3}+\gamma_{12} Y_{2}^{p+3}+\phi_{12}(\widehat{\theta}) z_{1}^{p+3}
\end{aligned}
$$

where $\gamma_{11}=\sum_{j=0}^{\left(p_{1}-1\right) / 2} \gamma_{11 j}, \gamma_{12}=\sum_{j=0}^{\left(p_{1}-1\right) / 2} \gamma_{12 j}$ with $\gamma_{11 j}$ and $\gamma_{12}$ being positive design parameters, $\phi_{20}(\hat{\theta}) \geq$ $\left(\left(p_{1}-2 j\right) /(p+3)\right)\left(\left((p+3) /\left(p-p_{1}+3+\right.\right.\right.$ $\left.2 j)) \gamma_{11 j}\right)^{-\left(p-p_{1}+3+2 j\right) /\left(p_{1}-2 j\right)}\left(k_{1} 2^{p_{1}-1-2 j} C_{p_{1}}^{2 j} \sqrt{1+\beta_{1}^{4 j}}\right)^{(p+3) /\left(p_{1}-2 j\right)}$, 
and $\phi_{12}(\hat{\theta}) \geq\left(p-p_{1}+3+2 j\right) /(p+3)$. $\left(\left((p+3) /\left(p_{1}-2 j\right)\right) \gamma_{12 j}\right)^{-\left(p_{1}-2 j\right) /\left(p-p_{1}+3+2 j\right)}\left(k_{1} 2^{p_{1}-1-2 j} C_{p_{1}}^{2 j}\right.$. $\left.\sqrt{1+\beta_{1}^{4 j}}\right)^{(p+3) /\left(p-p_{1}+3+2 j\right)}$. Substituting (16) into (15), one gets

$$
\begin{aligned}
\mathscr{L} V_{1} \leq & -\left(c_{1}-\gamma_{11}\right) z_{1}^{p+3}-v_{1}(\cdot) z_{1}^{p+3}+\phi_{20}(\widehat{\theta}) z_{2}^{p+3} \\
& +\gamma_{12} Y_{2}^{p+3}-\frac{1}{\Gamma} \tilde{\theta}\left(\dot{\hat{\theta}}-\tau_{1}\right)+\Delta_{1} \\
& +y^{p+3}\left(t-d_{1}(t)\right) S_{1}\left(y\left(t-d_{1}(t)\right)\right) .
\end{aligned}
$$

Step $i(2 \leq i \leq n-1)$. A similar result to Step 1 is obtained at this step. We state it in the following proposition.

Proposition 11. For the ith Lyapunov function

$$
V_{i}(\cdot)=\sum_{j=1}^{i} \frac{k_{j}}{p-p_{j}+4} z_{j}^{p-p_{j}+4}+\sum_{j=2}^{i} \frac{1}{p+3} Y_{j}^{p+3}+\frac{1}{2 \Gamma} \widetilde{\theta},
$$

there exists a virtual control law in the following form:

$$
\begin{aligned}
\alpha_{i+1}\left(\bar{z}_{i}, \widehat{\theta}\right) & =-z_{i}\left(\frac{c_{i}+\phi_{i 0}(\hat{\theta})+\phi_{i 1}(\widehat{\theta})}{k_{i}}\right)^{1 / p_{i}} \\
& =-z_{i} \beta_{i}(\widehat{\theta}),
\end{aligned}
$$

such that

$$
\begin{aligned}
\mathscr{L} V_{i} \leq & -\sum_{j=1}^{i}\left(c_{j}-\gamma_{j 1}\right) z_{j}^{p+3}-v_{1}(\cdot) z_{1}^{p+3} \\
& +\phi_{i+1,0}(\widehat{\theta}) z_{i+1}^{p+3}+\sum_{j=1}^{i} \gamma_{j 2} Y_{j+1}^{p+3}-\frac{1}{\Gamma} \widetilde{\theta}\left(\dot{\vec{\theta}}-\tau_{i}\right) \\
& +\Delta_{i}+\sum_{j=1}^{i} y^{p+3}\left(t-d_{j}(t)\right) S_{j}\left(y\left(t-d_{j}(t)\right)\right) \\
& +\sum_{j=1}^{i-1} Y_{j+1}^{p+2}\left(-\frac{Y_{j+1}}{q_{j+1}}+D_{j+1}\right),
\end{aligned}
$$

where $c_{i}, k_{i}$, and $\gamma_{j 1}$ are positive design constants, $\varphi_{i}(\hat{\theta})=$ $k_{i}^{2}+\sqrt{1+\widehat{\theta}^{2}} / 2+\varepsilon_{i}^{2} / 2, \tau_{i}=\sum_{j=1}^{i}(\Gamma / 2) z_{j}^{p-p_{j}+3}, \phi_{i 1}(\widehat{\theta}) \geq$ $\left(\left(\left(p-p_{i}+3\right) /(p+3)\right) \varphi_{i}(\widehat{\theta})\right)^{(p+3) /\left(p-p_{i}+3\right)}\left(p_{i} /\left(p-p_{i}+\right.\right.$ 3) $\left.\xi_{i}\right)^{p_{i} /\left(p-p_{i}+3\right)}, \Delta_{i}=\sum_{j=1}^{i}\left(\xi_{j 0}+\xi_{j 1}+\xi_{j 2}+\xi_{j}\right), \xi_{10}=0$, $\phi_{i 0}(\widehat{\theta}) \geq\left(\left(p_{i-1}-2 j\right) /(p+3)\right)\left((p+3) \gamma_{i-1,1 j} /(p-\right.$ $\left.\left.p_{i-1}+3+2 j\right)\right)^{-\left(p-p_{i-1}+3+2 j\right) /\left(p_{i-1}-2 j\right)}\left(k_{i-1} 2^{p_{i-1}-1-2 j} C_{p_{i-1}}^{2 j}\right.$. $\left.\sqrt{1+\beta_{i-1}^{4 j}}\right)^{(p+3) /\left(p_{i-1}-2 j\right)}$, and $S_{j}(\cdot)=\bar{\xi}_{j 1} \bar{f}_{j 2}^{p+3}(\cdot)+\bar{\xi}_{j 2} \bar{g}_{j 2}^{p+3}(\cdot)$.

Proof. See the Appendix.

Hence, at step $n$, we choose

$$
V_{n}(\cdot)=\sum_{i=1}^{n} \frac{k_{i}}{p-p_{i}+4} z_{i}^{p-p_{i}+4}+\sum_{i=2}^{n} \frac{1}{p+3} Y_{i}^{p+3}+\frac{1}{2 \Gamma} \widetilde{\theta} .
$$

By exactly following the design procedure in Proposition 11 and introducing Nussbaum function, one can construct the adaptive control laws as

$$
\begin{aligned}
u & =z_{n} N(\zeta) \beta_{n}(\widehat{\theta}), \\
\dot{\zeta} & =z_{n}^{p+3} \beta_{n}^{p_{n}}(\widehat{\theta}), \\
\beta_{n}(\widehat{\theta}) & =\left(c_{n}+\phi_{n 0}(\widehat{\theta})+\phi_{n 1}(\widehat{\theta})\right)^{1 / p_{n}}, \\
\dot{\hat{\theta}} & =\sum_{i=1}^{n} \frac{\Gamma}{2} z_{i}^{p-p_{i}+3}-k_{0} \widehat{\theta} ;
\end{aligned}
$$

thus, this leads to

$$
\begin{aligned}
\mathscr{L} V_{n} \leq & -\sum_{i=1}^{n}\left(c_{i}-\gamma_{i 1}\right) z_{i}^{p+3}-\gamma_{1}(\cdot) z_{1}^{p+3} \\
& +\sum_{i=1}^{n-1}\left(\gamma_{i 2}-\frac{1}{q_{i+1}}\right) Y_{i+1}^{p+3}+\sum_{i=1}^{n-1} Y_{i+1}^{p+2} D_{i+1}+\Delta_{n} \\
& +\frac{k_{0}}{\Gamma} \tilde{\theta} \widehat{\theta} \\
& +\sum_{i=1}^{n} y^{p+3}\left(t-d_{i}(t)\right) S_{i}\left(y\left(t-d_{i}(t)\right)\right) \\
& +k_{n} \eta(t) N^{p_{n}}(\zeta) \dot{\zeta}+\dot{\zeta},
\end{aligned}
$$

where $c_{n}, k_{0}>0, \gamma_{n 1}=0$ are design constants, $\varphi_{n}(\hat{\theta})=$ $k_{n}^{2}+\sqrt{1+\widehat{\theta}^{2}} / 2+\varepsilon_{n}^{2} / 2, \Delta_{n}=\sum_{i=1}^{n}\left(\xi_{i 0}+\xi_{i 1}+\xi_{i 2}+\right.$ $\left.\xi_{i}\right), \phi_{n 0}(\hat{\theta}) \geq\left(\left(p_{n-1}-2 j\right) /(p+3)\right)\left((p+3) \gamma_{n-1,1 j} /(p-\right.$ $\left.\left.p_{n-1}+3+2 j\right)\right)^{-\left(p-p_{n-1}+3+2 j\right) /\left(p_{n-1}-2 j\right)}\left(k_{n-1} 2^{p_{n-1}-1-2 j} C_{p_{n-1}}^{2 j}\right.$. $\left.\sqrt{1+\beta_{n-1}^{4 j}}\right)^{(p+3) /\left(p_{n-1}-2 j\right)}, \phi_{n 1}(\widehat{\theta}) \geq\left(\left(p-p_{n}+3\right) \varphi_{n}(\widehat{\theta}) /(p+\right.$ $3))^{(p+3) /\left(p-p_{n}+3\right)}\left(p_{n} /\left(p-p_{n}+3\right) \xi_{n}\right)^{p_{n} /\left(p-p_{n}+3\right)}, \xi_{n 0}=0$, and $S_{i}\left(y\left(t-d_{i}(t)\right)\right)=\bar{\xi}_{i 1} \bar{f}_{i 2}^{p+3}\left(y\left(t-d_{i}(t)\right)\right)+\bar{\xi}_{i 2} \bar{g}_{i 2}^{p+3}\left(y\left(t-d_{i}(t)\right)\right)$.

Remark 12. During the design procedure, the drift and diffusion terms are technically handled for more general system (1) by RBF NN approximation approach compared with $[29,30,32-34]$. Furthermore, the repeated differentiation of virtual control laws is avoided and the unknown time-varying control direction is successfully handled by introducing DSC and Nussbaum-type gain function.

\section{Stability Analysis}

The main result of this paper is stated in the following theorem.

Theorem 13. For stochastic high-order time-delay system (1) satisfying Assumptions 7-9, when the adaptive control laws are chosen as (14), (19), and (22) and the initial condition satisfies $V(0) \leq \sigma$, the closed-loop system consisting of (1), (8), (14), (19), and (22) is semiglobally uniformly ultimately bounded (SGUUB), where $V$ is the closed-loop Lyapunov-Krasovskii functional and $\sigma$ is some constant. 
Proof. Choosing Lyapunov-Krasovskii functional $V=V_{n}+W$ as

$$
V=V_{n}+\sum_{i=1}^{n} \frac{1}{1-\gamma_{i}} \int_{t-d_{i}(t)}^{t} y^{p+3} S_{i}(y(s)) d s,
$$

in terms of Assumption 7 and (23)-(24), one obtains

$$
\begin{aligned}
\mathscr{L} V \leq & -\sum_{i=1}^{n}\left(c_{i}-\gamma_{i 1}\right) z_{i}^{p+3}-v_{1}(\cdot) z_{1}^{p+3} \\
& +\sum_{i=1}^{n} \frac{1}{1-\gamma_{i}} y^{p+3} S_{i}(y)+\sum_{i=1}^{n-1}\left(\gamma_{i 2}-\frac{1}{q_{i+1}}\right) Y_{i+1}^{p+3} \\
& +\sum_{i=1}^{n-1} Y_{i+1}^{p+2} D_{i+1}+\Delta_{n}+\frac{k_{0}}{\Gamma} \widetilde{\theta} \widehat{\theta} \\
& +k_{n} \eta(t) N^{p_{n}}(\zeta) \dot{\zeta}+\dot{\zeta} .
\end{aligned}
$$

Let $\nu_{1}(\cdot)-\sum_{i=1}^{n}\left(1 /\left(1-\gamma_{i}\right)\right) S_{i}(y) \geq 0$ and use Lemma 3 to estimate the terms in (25) as

$$
\begin{aligned}
Y_{i+1}^{p+2} D_{i+1} \leq & \frac{p+2}{p+3}\left(\varepsilon_{0} D_{i+1}\right)^{(p+3) /(p+2)} Y_{i+1}^{p+3} \\
& +\frac{1}{(p+3) \varepsilon_{0}^{p+3}}, \\
\tilde{\theta} \hat{\theta} \leq & -\frac{1}{2} \widetilde{\theta}^{2}+\frac{1}{2} \theta^{2}
\end{aligned}
$$

one can get

$$
\begin{aligned}
\mathscr{L} V & \leq-\sum_{i=1}^{n}\left(c_{i}-\gamma_{i 1}\right) z_{i}^{p+3} \\
& -\sum_{i=1}^{n-1}\left(\frac{1}{q_{i+1}}-\gamma_{i 2}-\frac{p+2}{p+3}\left(\varepsilon_{0} D_{i+1}\right)^{(p+3) /(p+2)}\right) Y_{i+1}^{p+3} \\
& -\frac{k_{0}}{2 \Gamma} \widetilde{\theta}^{2}+\Delta_{n+1}+k_{n} \eta(t) N^{p_{n}}(\zeta) \dot{\zeta}+\dot{\zeta}
\end{aligned}
$$

where $\varepsilon_{0}>0$ is a parameter and $\Delta_{n+1}=\Delta_{n}+1 /(p+3) \varepsilon_{0}^{p+3}+$ $\left(k_{0} / 2 \Gamma\right) \theta^{2}$.

By estimating $V_{n}$ as $V-W \leq \sum_{i=1}^{n} \rho_{i} z_{i}^{p+3}+\Delta_{0}+\sum_{i=1}^{n-1}(1 /(p+$ 3)) $Y_{i+1}^{p+3}+(1 / 2 \Gamma) \widetilde{\theta}^{2}$ and choosing

$$
\begin{aligned}
\bar{\lambda} & =\min \left\{\frac{c_{i}-\gamma_{i 1}}{\rho_{i}},(p+3)\right. \\
& \left.\cdot\left(\frac{1}{q_{i+1}}-\gamma_{i 2}-\frac{p+2}{p+3}\left(\varepsilon_{0} D_{i+1}\right)^{(p+3) /(p+2)}\right), k_{0}, 1\right\}, \\
\mu & =\Delta_{n+1}+\bar{\lambda} \Delta_{0}
\end{aligned}
$$

this leads to

$$
\begin{aligned}
\mathscr{L} V & \leq-\bar{\lambda}(V-W)+\mu+k_{n} \eta(t) N^{p_{n}}(\zeta) \dot{\zeta}+\dot{\zeta} \\
& \leq-\lambda V+\mu+k_{n} \eta(t) N^{p_{n}}(\zeta) \dot{\zeta}+\dot{\zeta}
\end{aligned}
$$

where $\Delta_{0}$ is a design constant, $\rho_{i}=\left(\left(p-p_{i}+4\right) k_{i} /(p+\right.$ $3))^{(p+3) /\left(p-p_{i}+4\right)}\left(\left(p_{i}-1\right) /\left(p-p_{i}+4\right) \Delta_{0}\right)^{\left(p_{i}-1\right) /\left(p-p_{i}+4\right)}$, and $\lambda$ is a positive parameter to be chosen.

Multiplying (29) by $e^{\lambda t}$, taking expectations on both sides and integrating it over $[0, t]$, one has

$$
\begin{aligned}
E\left(e^{\lambda t} V\right) \leq & E(V(0))+\frac{\mu}{\lambda}\left(e^{\lambda t}-1\right) \\
& +\int_{0}^{t}\left(k_{n} \eta(s) N^{p_{n}}(\zeta)+1\right) \dot{\zeta} e^{\lambda s} d s
\end{aligned}
$$

then,

$$
\begin{aligned}
E(V) \leq & E(V(0)) e^{-\lambda t}+\frac{\mu}{\lambda}\left(1-e^{-\lambda t}\right) \\
& +e^{-\lambda t} \int_{0}^{t}\left(k_{n} \eta(s) N^{p_{n}}(\zeta)+1\right) \dot{\zeta} e^{\lambda s} d s \\
\leq & E(V(0)) e^{-\lambda t}+\frac{\mu}{\lambda} \\
& +\int_{0}^{t}\left(k_{n} \eta(s) N^{p_{n}}(\zeta)+1\right) \dot{\zeta} e^{-\lambda(t-s)} d s .
\end{aligned}
$$

It is easy to see that, in (31), $0<e^{-\lambda(t-s)} \leq 1$ for $s \in[0, t]$. Let $e^{-\lambda(t-s)} N^{p_{n}}(\zeta)$ be a new Nussbaum function; according to Definition 1, one has $\lim _{\zeta \rightarrow \infty}$ sup $\int e^{-\lambda(t-s)} N^{p_{n}}(\zeta) \dot{\zeta} \rightarrow+\infty$ and $\lim _{\zeta \rightarrow \infty} \inf \int e^{-\lambda(t-s)} N^{p_{n}}(\zeta) \dot{\zeta} \rightarrow-\infty$. Then, it can be obtained that $E(V)<0$, but this counteracts the fact that $E(\mathrm{~V}) \geq 0$; therefore, $\zeta$ and $e^{-\lambda(t-s)} N^{p_{n}}(\zeta) \dot{\zeta}$ must be bounded by some constant, which shows that $E(V)$ must be bounded to some constant.

From the above discussion and Definition 2, one gets that $V$ is SGUUB. Thus, from the forms of $V$ and $V_{i}$ in the design procedure, one concludes that all the variables in the closedsystem are SGUUB; that is to say the closed-loop system consisting of (1), (8), (14), (19), and (22) is SGUUB. The proof is completed.

\section{A Simulation Example}

Consider the following stochastic high-order nonlinear system:

$$
\begin{aligned}
d x_{1} & =x_{2}^{3} d t+f_{1}(\cdot) d t+g_{1}(\cdot) d \omega \\
d x_{2} & =\eta(t) x_{2}^{5} d t+f_{2}(\cdot) d t+g_{2}(\cdot) d \omega \\
y & =x_{1}
\end{aligned}
$$

where $p=\max \{3,5\}=5, f_{1}=x_{1}^{3} /\left(1+x_{1}^{2}\right)+\left(x_{1}(t-\right.$ $\left.\left.d_{1}(t)\right)\right) \sin \left(x_{2}\right), g_{1}=x_{1}^{2} x_{1}\left(t-d_{1}(t)\right), f_{2}=x_{1} \cos \left(x_{2}\right)+$ $(1 / 5) x_{1} x_{1}^{2}\left(t-d_{1}(t)\right), g_{2}=x_{1}^{2}\left(t-d_{1}(t)\right) /\left(1+x_{2}^{2}\right)+x_{1}^{2} /\left(1+x_{1}^{2}\right)$, $\eta(t)=1+0.1 \sin (t), d_{1}(t)=(1 / 4)(1+\sin (t))$, and $d_{2}(t)=$ $(1 / 5)(1+\sin (t))$. It can be verified that Assumptions $7-9$ hold true. 

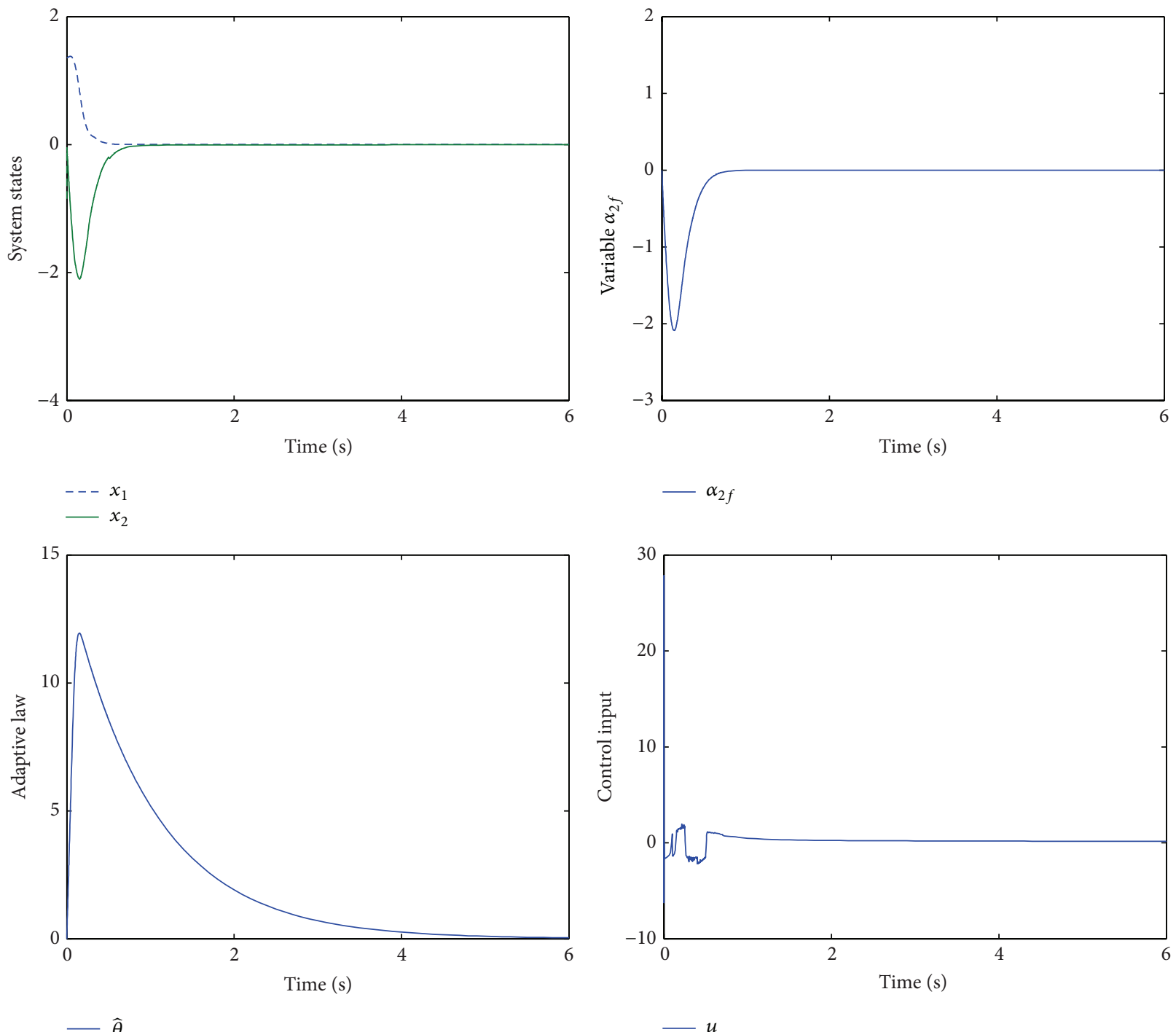

Figure 1: The responses of the closed-loop system (32)-(33).

By exactly following the design procedure in Section 3, we construct the adaptive control laws

$$
\begin{aligned}
z_{1} & =x_{1} \\
z_{2} & =x_{2}-\alpha_{2 f}, \\
\alpha_{2} & =-z_{1}\left(\frac{c_{1}+\phi_{11}(\widehat{\theta})}{k_{1}}\right)^{1 / 3}=-z_{1} \beta_{1}(\widehat{\theta}), \\
u & =z_{2} N(\zeta) \beta_{2}(\widehat{\theta}) \\
\dot{\zeta} & =z_{2}^{8} \beta_{2}^{5}(\widehat{\theta}) \\
\beta_{2} & =\left(c_{2}+\phi_{20}(\widehat{\theta})+\phi_{21}(\widehat{\theta})\right)^{1 / 5} \\
\dot{\hat{\theta}} & =\frac{\Gamma}{2}\left(z_{1}^{5}+z_{2}^{3}\right)-k_{0} \hat{\theta}
\end{aligned}
$$

where $c_{1}, c_{2}, k_{0}, k_{1}, k_{2}$, and $\Gamma$ are positive design constants, $q_{2} \dot{\alpha}_{2 f}+\alpha_{2 f}=\alpha_{2}$ with $q_{2}>0$ being a time constant of a filter, $\varphi_{1}(\hat{\theta})=k_{1}^{2}+\sqrt{1+\widehat{\theta}^{2}} / 2+\varepsilon_{1}^{2} / 2$, $\phi_{11}=\left((5 / 8) \varphi_{1}(\widehat{\theta})\right)^{8 / 5}\left(3 / 5 \xi_{1}\right)^{3 / 5}, \varphi_{2}(\widehat{\theta})=k_{2}^{2}+\sqrt{1+\widehat{\theta}^{2}} / 2+$ $\varepsilon_{2}^{2} / 2, \phi_{20}=(3 / 8)\left((8 / 5) \gamma_{110}\right)^{-5 / 3}\left((\sqrt{2} / 4) k_{1}\right)^{8 / 3}+(1 / 8)((8 /$ 7) $\left.\gamma_{111}\right)^{-7}\left(3 k_{1} \sqrt{1+\beta_{1}^{4}}\right)^{8}, \phi_{21}=\left((3 / 8) \varphi_{2}(\widehat{\theta})\right)^{8 / 3}\left(3 / 5 \xi_{2}\right)^{3 / 5}$, and $\varepsilon_{1}, \varepsilon_{2}, \xi_{1}, \xi_{2}, \gamma_{110}$, and $\gamma_{111}$ are positive parameters.

In simulation, choose the Nussbaum function as $N(\zeta)=$ $e^{\zeta^{2}} \cos ((\pi / 2) \zeta)$ and let the design constants and parameters be $c_{1}=12.5, c_{2}=5, k_{0}=1, k_{1}=2, k_{2}=0.5, \Gamma=50, \varepsilon_{1}=0.01$, $\varepsilon_{2}=0.01, \xi_{1}=1, \xi_{2}=1, \gamma_{110}=2, \gamma_{111}=2$, and $q_{2}=0.1$. The initial conditions are given by $x_{1}(0)=1.35, x_{2}(0)=-0.85$, $\hat{\theta}(0)=0$, and $\zeta(0)=0$. From Figure 1 , one can see that system states, control input, adaptive law, and variable $\alpha_{2 f}$ converge to the origin uniformly, which means that all the signals of the closed-loop (32)-(33) are SGUUB. Figure 1 demonstrates the effectiveness of the control scheme. 


\section{Conclusions}

This paper considers the unknown time-varying control coefficient by generalizing Nussbaum function to a class of stochastic high-order time-delay systems. Under weaker conditions on the drift and diffusion terms, the adaptive statefeedback control is solved by using RBF NN approximation approach. The control scheme ensures the closed-loop system to be SGUUB. An issue to be investigated is how to further handle the output-feedback problem for system (1).

\section{Appendix}

Proof of Proposition 11. We prove this proposition by the frequently used induction method. Assume that, at step $(i-1)$, there exist a series of virtual control laws

$$
\begin{aligned}
\alpha_{j+1}\left(\bar{z}_{j}, \widehat{\theta}\right) & =-z_{j}\left(\frac{c_{j}+\phi_{j 0}(\widehat{\theta})+\phi_{j 1}(\widehat{\theta})}{k_{j}}\right)^{1 / p_{j}} \\
& =-z_{j} \beta_{j}(\hat{\theta}), \quad j=2, \ldots, i-1,
\end{aligned}
$$

such that

$$
\begin{aligned}
\mathscr{L} V_{i-1} \leq & -\sum_{j=1}^{i-1}\left(c_{j}-\gamma_{j 1}\right) z_{j}^{p+3}-v_{1}(\cdot) z_{1}^{p+3} \\
& +\phi_{i 0}(\widehat{\theta}) z_{i}^{p+3}+\sum_{j=1}^{i-1} \gamma_{j 2} Y_{j+1}^{p+3} \\
& -\frac{1}{\Gamma} \widetilde{\theta}\left(\dot{\hat{\theta}}-\tau_{i-1}\right)+\Delta_{i-1} \\
& +\sum_{j=1}^{i-1} y^{p+3}\left(t-d_{j}(t)\right) S_{j}\left(y\left(t-d_{j}(t)\right)\right) \\
& +\sum_{j=1}^{i-2} Y_{j+1}^{p+2}\left(-\frac{Y_{j+1}}{q_{j+1}}+D_{j+1}\right)
\end{aligned}
$$

holds for $V_{i-1}=\sum_{j=1}^{i-1}\left(k_{j} /\left(p-p_{j}+4\right)\right) z_{j}^{p-p_{j}+4}+\sum_{j=2}^{i-1}(1 /(p+$ 3)) $Y_{j}^{p+3}+(1 / 2 \Gamma) \tilde{\theta}$, where the parameters and functions are designed as in Proposition 11.

In the sequel, we prove that (A.2) still holds for (18). By using (3), (8), (18), (A.2), and Itŏ's formula, one has

$$
\begin{aligned}
\mathscr{L} V_{i} \leq & -\sum_{j=1}^{i-1}\left(c_{j}-\gamma_{j 1}\right) z_{j}^{p+3}-v_{1}(\cdot) z_{1}^{p+3} \\
& +\phi_{i 0}(\widehat{\theta}) z_{i}^{p+3}+\sum_{j=1}^{i-1} \gamma_{j 2} Y_{j+1}^{p+3}-\frac{1}{\Gamma} \widetilde{\theta}\left(\dot{\hat{\theta}}-\tau_{i-1}\right) \\
& +\Delta_{i-1} \\
& +\sum_{j=1}^{i-1} y^{p+3}\left(t-d_{j}(t)\right) S_{j}\left(y\left(t-d_{j}(t)\right)\right)
\end{aligned}
$$

$$
\begin{aligned}
& +\sum_{j=1}^{i-1} Y_{j+1}^{p+2}\left(-\frac{Y_{j+1}}{q_{j+1}}+D_{j+1}\right) \\
& +k_{i} z_{i}^{p-p_{i}+3}\left(x_{i+1}^{p_{i}}+f_{i}(\cdot)-\dot{\alpha}_{i f}\right) \\
& +\frac{p-p_{i}+3}{2} k_{i} z_{i}^{p-p_{i}+2} g_{i}(\cdot) g_{i}^{T}(\cdot) .
\end{aligned}
$$

Estimate the terms on the right-hand side of (A.3) with the help of Lemmas 4-5 and Assumption 9 as

$$
\begin{aligned}
k_{i} z_{i}^{p-p_{i}+3}\left(f_{i}(\cdot)-\dot{\alpha}_{i f}\right) \leq k_{i} z_{i}^{p-p_{i}+3}\left(f_{i 1}(y)-\dot{\alpha}_{i f}\right) \\
\quad+l_{i 1} z_{i}^{p-p_{i}+4}+\bar{l}_{i 1} f_{i 2}^{p-p_{i}+4}\left(y\left(t-d_{i}(t)\right)\right) \\
\quad \leq k_{i} z_{i}^{p-p_{i}+3}\left(f_{i 1}(y)-\dot{\alpha}_{i f}\right)+l_{i 1} z_{i}^{p-p_{i}+4}+\xi_{i 1} \\
+\bar{\xi}_{i 1} f_{i 2}^{p+3}\left(y\left(t-d_{i}(t)\right)\right), \\
\frac{p-}{p_{i}+3} k_{i} z_{i}^{p-p_{i}+2} g_{i}(\cdot) g_{i}^{T}(\cdot) \leq k_{i}\left(p-p_{i}+3\right) \\
\quad 2 \\
\quad z_{i}^{p-p_{i}+2}\left(g_{i 1}^{2}(y)+g_{i 2}^{2}\left(y\left(t-d_{i}(t)\right)\right)\right) \leq \xi_{i 0} \\
+\rho(y) z_{i}^{p-p_{i}+3}+l_{i 2} z_{i}^{p-p_{i}+4} \\
+\bar{l}_{i 2} g_{i 2}^{p-p_{i}+4}\left(y\left(t-d_{i}(t)\right)\right) \leq \xi_{i 0}+\rho(y) z_{i}^{p-p_{i}+3} \\
+l_{i 2} z_{i}^{p-p_{i}+4}+\xi_{i 2}+\bar{\xi}_{i 2} g_{i 2}^{p+3}\left(y\left(t-d_{i}(t)\right)\right),
\end{aligned}
$$

where $l_{i 1}, l_{i 2}, \xi_{i 0}, \xi_{i 1}$, and $\xi_{i 2}>0$ are parameters, $\bar{l}_{i 1}=$ $\left(1 /\left(p-p_{i}+4\right)\right)\left(\left(\left(p-p_{i}+4\right) /\left(p-p_{i}+3\right)\right) l_{i 1}\right)^{-\left(p-p_{i}+3\right)} k_{i}^{p-p_{i}+4}$, $\bar{\xi}_{i 1}=\left(\left(\left(p-p_{i}+4\right) /(p+3)\right) \bar{l}_{i 1}\right)^{(p+3) /\left(p-p_{i}+4\right)}\left(\left(p_{i}-1\right) /\left(p-p_{i}+\right.\right.$ $\left.4) \xi_{i 1}\right)^{\left(p_{i}-1\right) /\left(p-p_{i}+4\right)}, \bar{\xi}_{i 0}=\left(\left(\left(p-p_{i}+2\right) /\left(p-p_{i}+3\right)\right) k_{i}(p-\right.$ $\left.\left.p_{i}+3\right)\right)^{\left(p-p_{i}+3\right) /\left(p-p_{i}+2\right)}\left(1 /\left(p-p_{i}+2\right) \xi_{i 0}\right)^{1 /\left(p-p_{i}+2\right)}, \bar{l}_{i 2}=(2 /(p-$ $\left.\left.p_{i}+4\right)\right)\left(\left(\left(p-p_{i}+4\right) /\left(p-p_{i}+2\right)\right) l_{i 2}\right)^{-\left(p-p_{i}+2\right) / 2}\left(k_{i}\left(p-p_{i}+\right.\right.$ $3))^{\left(p-p_{i}+4\right) / 2}, \bar{\xi}_{i 2}=\left(\left(\left(p-p_{i}+4\right) /(p+3)\right) \bar{l}_{i 2}\right)^{(p+3) /\left(p-p_{i}+4\right)}\left(\left(p_{i}-\right.\right.$ $\left.1) /\left(p-p_{i}+4\right) \xi_{i 2}\right)^{\left(p_{i}-1\right) /\left(p-p_{i}+4\right)}$ are design constants, and $\rho(y)=$ $\bar{\xi}_{i 0} g_{i 1}^{2\left(p-p_{i}+3\right) /\left(p-p_{i}+2\right)}(y)$.

Then, substituting (A.4) into (A.3) and constructing the intermediate variable as

$$
\begin{aligned}
& \widehat{\alpha}_{i+1, f}^{p_{i}}\left(X_{i}\right) \\
& =-\frac{z_{i}\left(l_{i 1}+l_{i 2}\right)+k_{i}\left(f_{i 1}-\dot{\alpha}_{i f}\right)+\rho(y)+\phi_{i 2}(\widehat{\theta}) z_{i}^{p_{i}}}{k_{i}},
\end{aligned}
$$

one gets

$$
\begin{aligned}
\mathscr{L} V_{i} \leq & -\sum_{j=1}^{i-1}\left(c_{j}-\gamma_{j 1}\right) z_{j}^{p+3}-v_{1}(\cdot) z_{1}^{p+3} \\
& +\phi_{i 0}(\widehat{\theta}) z_{i}^{p+3}-\phi_{i 2}(\widehat{\theta}) z_{i}^{p+3}+\sum_{j=1}^{i-1} \gamma_{j 2} Y_{j+1}^{p+3} \\
& +\Delta_{i-1}+\xi_{i 0}+\xi_{i 1}+\xi_{i 2}-\frac{1}{\Gamma} \tilde{\theta}\left(\dot{\hat{\theta}}-\tau_{i-1}\right)
\end{aligned}
$$




$$
\begin{aligned}
& +\sum_{j=1}^{i} y^{p+3}\left(t-d_{j}(t)\right) S_{j}\left(y\left(t-d_{j}(t)\right)\right) \\
& +\sum_{j=1}^{i-1} Y_{j+1}^{p+2}\left(-\frac{Y_{j+1}}{q_{j+1}}+D_{j+1}\right) \\
& +k_{i} z_{i}^{p-p_{i}+3}\left(x_{i+1}^{p_{i}}-\widehat{\alpha}_{i+1, f}^{p_{i}}\right),
\end{aligned}
$$

where $X_{i}=\left(\bar{z}_{i}, \dot{\alpha}_{i f}, \bar{\alpha}_{i f}\right)^{T}$ and $\phi_{i 2}(\widehat{\theta})$ is a design function.

Utilizing Lemmas 3-4 and RBF NN (5), there exists $W_{i}^{*^{*}} S_{i}$ such that $\hat{\alpha}_{i+1, f}^{p_{i}}=W_{i}^{*^{T}} S_{i}+\delta_{i},\left|\delta_{i}\right|<\varepsilon_{i}$. Thus, we have

$$
\begin{aligned}
-k_{i} z_{i}^{p-p_{i}+3} \widehat{\alpha}_{i+1, f}^{p_{i}} & \leq z_{i}^{p-p_{i}+3}\left(k_{i}^{2}+\frac{\theta}{2}+\frac{\varepsilon_{i}^{2}}{2}\right) \\
& \leq z_{i}^{p-p_{i}+3} \varphi_{i 1}(\widehat{\theta})+\frac{1}{2} z_{i}^{p-p_{i}+3} \widetilde{\theta} \\
& \leq \xi_{i}+z_{i}^{p+3} \phi_{i 1}(\widehat{\theta})+\frac{1}{2} z_{i}^{p-p_{i}+3} \widetilde{\theta}
\end{aligned}
$$

where $\xi_{i}>0,\left|W_{i}^{*^{T}}\right|^{2} S_{i}^{2} \leq\left|W_{i}^{*^{T}}\right|^{2} N_{i} \leq \theta, \phi_{i 1} \geq((p-$ $\left.\left.p_{i}+3\right) \varphi_{i 1}(\widehat{\theta}) /(p+3)\right)^{(p+3) /\left(p-p_{i}+3\right)}\left(p_{i} /\left(p-p_{i}+3\right) \xi_{i}\right)^{p_{i} /\left(p-p_{i}+3\right)}$, and $\varphi_{i 1}(\widehat{\theta})=k_{i}^{2}+\sqrt{1+\widehat{\theta}^{2}} / 2+\varepsilon_{i}^{2} / 2$. By applying (A.7) and constructing the $i$ th virtual control law as (19), one arrives at

$$
\begin{aligned}
\mathscr{L} V_{i} \leq & -\sum_{j=1}^{i-1}\left(c_{j}-\gamma_{j 1}\right) z_{j}^{p+3}-c_{i} z_{i}^{p+3}-v_{1}(\cdot) z_{1}^{p+3} \\
& -\phi_{i 2}(\widehat{\theta}) z_{i}^{p+3}+\sum_{j=1}^{i-1} \gamma_{j 2} Y_{j+1}^{p+3} \\
& +\sum_{j=1}^{i} y^{p+3}\left(t-d_{j}(t)\right) S_{j}\left(y\left(t-d_{j}(t)\right)\right) \\
& +\sum_{j=1}^{i-1} Y_{j+1}^{p+2}\left(-\frac{Y_{j+1}}{q_{j+1}}+D_{j+1}\right) \\
& +k_{i} z_{i}^{p-p_{i}+3}\left(x_{i+1}^{p_{i}}-\alpha_{i+1}^{p_{i}}\right)+\Delta_{i} \\
& -\frac{1}{\Gamma} \widetilde{\theta}\left(\dot{\hat{\theta}}-\tau_{i}\right)
\end{aligned}
$$

where $\Delta_{i}=\Delta_{i-1}+\xi_{i 0}+\xi_{i 1}+\xi_{i 2}+\xi_{i}, \tau_{i}=\sum_{j=1}^{i}(\Gamma / 2) z_{j}^{p-p_{j}+3}$. Now, let $\alpha_{i+1, f}$ be obtained by a first-order filter with time constant $q_{i+1}$; then, it holds that $q_{i+1} \dot{\alpha}_{i+1, f}+\alpha_{i+1, f}=\alpha_{i+1}$ and $\alpha_{i+1, f}(0)=\alpha_{i+1}(0)$. Define $Y_{i+1}=\alpha_{i+1, f}-\alpha_{i+1}$ as the output error; it is obvious that $\dot{\alpha}_{i+1, f}=-Y_{i+1} / q_{i+1}$ and $\dot{Y}_{i+1}=$ $-Y_{i+1} / q_{i+1}+D_{i+1}\left(X_{i}\right)$, where $D_{i+1}\left(X_{i}\right)=\dot{z}_{i} \beta_{i}(\widehat{\theta})+z_{i} \dot{\beta_{i}}(\hat{\theta}) \dot{\hat{\theta}}$.
With the help of Lemmas 5-6 and $(a+b)^{n}=\sum_{i=0}^{n} C_{n}^{i} a^{n-i} b^{i}$, this yields

$$
\begin{aligned}
& k_{i} z_{i}^{p-p_{1}+3}\left(x_{i+1}^{p_{i}}-\alpha_{i+1}^{p_{i}}\right) \\
& \leq k_{i} z_{i}^{p-p_{i}+3}\left(\sum_{j=0}^{p_{i}-1} C_{p_{i}}^{j}\left(z_{i+1}+Y_{i+1}\right)^{p_{i}-j}\right. \\
& \left.\cdot\left(-z_{i} \beta_{i}(\widehat{\theta})\right)^{j}\right) \\
& \quad \leq k_{i} \sum_{j=0}^{\left(p_{i}-1\right) / 2} C_{p_{i}}^{2 j} 2^{p_{i}-1-2 j} z_{i}^{p-p_{i}+3+2 j}\left(\left|z_{i+1}\right|^{p_{i}-2 j}\right. \\
& \left.+\left|Y_{i+1}\right|^{p_{i}-2 j}\right) \beta_{i}^{2 j} \leq \gamma_{i 1} z_{i}^{p+3}+\phi_{i+1,0}(\widehat{\theta}) z_{i+1}^{p+3} \\
& +\gamma_{i 2} Y_{i+1}^{p+3}+\phi_{i, 2}(\widehat{\theta}) z_{i}^{p+3},
\end{aligned}
$$

where $\gamma_{i 1}=\sum_{j=0}^{\left(p_{i}-1\right) / 2} \gamma_{i 1 j}$ and $\gamma_{i 2}=\sum_{j=0}^{\left(p_{i}-1\right) / 2} \gamma_{i 2 j}$ with $\gamma_{i 1 j}$ and $\gamma_{i 2 j}$ being positive design parameters, $\phi_{i+1,0}(\cdot) \geq$ $\left(\left(p_{i}-2 j\right) /(p+3)\right)\left((p+3) \gamma_{i 1 j} /\left(p-p_{i}+3+\right.\right.$ $2 j))^{-\left(p-p_{i}+3+2 j\right) /\left(p_{i}-2 j\right)}\left(k_{i} 2^{p_{i}-1-2 j} C_{p_{i}}^{2 j} \sqrt{1+\beta_{i}^{4 j}}\right)^{(p+3) /\left(p_{i}-2 j\right)}$, and $\phi_{i 2}(\cdot) \geq\left(\left(p-p_{i}+3+2 j\right) /(p+3)\right)\left((p+3) /\left(p_{i}-2 j\right)\right.$. $\left.\gamma_{i 2 j}\right)^{-\left(p_{i}-2 j\right) /\left(p-p_{i}+3+2 j\right)}\left(k_{i} 2^{p_{i}-2 j-1} C_{p_{i}}^{2 j} \sqrt{1+\beta_{i}^{4 j}}\right)^{(p+3) /\left(p-p_{i}+3+2 j\right)}$. Substituting (A.9) into (A.8), one obtains (20). The proof is completed.

\section{Conflict of Interests}

The authors declare that there is no conflict of interests regarding the publication of this paper.

\section{Acknowledgments}

This work is supported by the National Natural Science Foundation of China (nos. 61573172, 61305149, and 61403174), 333 High-Level Talents Training Program in Jiangsu Province (no. BRA2015352), and Program for Fundamental Research of Natural Sciences in Universities of Jiangsu Province (no. 15KJB510011).

\section{References}

[1] M. Krstić and H. Deng, Stabilization of Uncertain Nonlinear Systems, Springer, New York, NY, USA, 1998.

[2] H. Deng, M. Krstić, and R. J. Williams, "Stabilization of stochastic nonlinear systems driven by noise of unknown covariance," IEEE Transactions on Automatic Control, vol. 46, no. 8, pp. 12371253, 2001.

[3] X. R. Mao, Stochastic Differential Equations and Their Applications, Horwood Publishing, Chichester, UK, 2007.

[4] H. E. Psillakis and A. T. Alexandridis, "NN-based adaptive tracking control of uncertain nonlinear systems disturbed by unknown covariance noise," IEEE Transactions on Neural Networks, vol. 18, no. 6, pp. 1830-1835, 2007. 
[5] W. S. Chen, L. C. Jiao, and Z. B. Du, "Output-feedback adaptive dynamic surface control of stochastic non-linear systems using neural network," IET Control Theory \& Applications, vol. 4, no. 12, pp. 3012-3021, 2010.

[6] W. S. Chen, "Output-feedback adaptive stochastic nonlinear stabilization using neural networks," Journal of Systems Engineering and Electronics, vol. 21, no. 1, pp. 81-87, 2010.

[7] T. Wang, S. C. Tong, and Y. M. Li, "Adaptive neural network output feedback control of stochastic nonlinear systems with dynamical uncertainties," Neural Computing and Applications, vol. 23, no. 5, pp. 1481-1494, 2013.

[8] H.-Q. Wang, B. Chen, and C. Lin, "Adaptive neural tracking control for a class of stochastic nonlinear systems," International Journal of Robust and Nonlinear Control, vol. 24, no. 7, pp. 12621280, 2014.

[9] H. Q. Wang, B. Chen, and C. Lin, "Adaptive neural control for strict-feedback stochastic nonlinear systems with time-delay," Neurocomputing, vol. 77, no. 1, pp. 267-274, 2012.

[10] H. T. Gao, T. P. Zhang, and X. N. Xia, "Adaptive neural control of stochastic nonlinear systems with unmodeled dynamics and time-varying state delays," Journal of the Franklin Institute, vol. 351, no. 6, pp. 3182-3199, 2014.

[11] Z. X. Yu, S. G. Li, and H. B. Du, "Adaptive neural output feedback control for stochastic nonlinear time-delay systems with unknown control directions," Neural Computing and Applications, vol. 25, no. 7-8, pp. 1979-1992, 2014.

[12] Z. X. Yu and S. G. Li, "Neural-network-based output-feedback adaptive dynamic surface control for a class of stochastic nonlinear time-delay systems with unknown control directions," Neurocomputing, vol. 129, pp. 540-547, 2014.

[13] Q. Zhou, P. Shi, Y. S. Xu, and Y. H. Li, "Observer-based adaptive neural network control for nonlinear stochastic systems with time delay," IEEE Transactions on Neural Networks and Learning Systems, vol. 24, no. 1, pp. 71-80, 2013.

[14] Z. X. Yu and H. B. Du, "Adaptive neural control for uncertain stochastic nonlinear strict-feedback systems with time-varying delays: a Razumikhin functional method," Neurocomputing, vol. 74, no. 12-13, pp. 2072-2082, 2011.

[15] Z. X. Yu, Z. H. Jin, and H. B. Du, "Adaptive neural control for a class of non-affine stochastic non-linear systems with timevarying delay: a Razumikhin-Nussbaum method," IET Control Theory \& Applications, vol. 6, no. 1, pp. 14-23, 2012.

[16] Z.-X. Yu and Z.-S. Yu, "Adaptive neural dynamic surface control for nonlinear pure-feedback systems with multiple time-varying delays: a Lyapunov-Razumikhin method," Asian Journal of Control, vol. 15, no. 4, pp. 1124-1138, 2013.

[17] G. Z. Cui, T. C. Jiao, Y. L. Wei, G. Song, and Y. Chu, "Adaptive neural control of stochastic nonlinear systems with multiple time-varying delays and input saturation," Neural Computing and Applications, vol. 25, no. 3-4, pp. 779-791, 2014.

[18] X.-J. Xie and J. Tian, "Adaptive state-feedback stabilization of high-order stochastic systems with nonlinear parameterization," Automatica, vol. 45, no. 1, pp. 126-133, 2009.

[19] W. Q. Li, Y. W. Jing, and S. Y. Zhang, "State feedback control of a class of high-order stochastic nonlinear systems," International Journal of Robust and Nonlinear Control, vol. 22, no. 14, pp. 15831601, 2012.

[20] J.-Y. Zhai and H.-B. Du, "Global output feedback stabilisation for a class of upper triangular stochastic nonlinear systems," International Journal of Control, vol. 87, no. 10, pp. 2106-2117, 2014.
[21] J.-Y. Zhai, "Decentralised output-feedback control for a class of stochastic non-linear systems using homogeneous domination approach," IET Control Theory \& Applications, vol. 7, no. 8, pp. 1098-1109, 2013.

[22] W. Q. Li, X.-J. Xie, and S. Y. Zhang, “Output-feedback stabilization of stochastic high-order nonlinear systems under weaker conditions," SIAM Journal on Control and Optimization, vol. 49, no. 3, pp. 1262-1282, 2011.

[23] C.-R. Zhao and X.-J. Xie, “Output feedback stabilization using small-gain method and reduced-order observer for stochastic nonlinear systems," IEEE Transactions on Automatic Control, vol. 58, no. 2, pp. 523-529, 2013.

[24] X.-J. Xie, N. Duan, and X. Yu, "State-feedback control of highorder stochastic nonlinear systems with SiISS inverse dynamics," IEEE Transactions on Automatic Control, vol. 56, no. 8, pp. 1921-1926, 2011.

[25] J.-Y. Zhai, "Finite-time output feedback stabilization for stochastic high-order nonlinear systems," Circuits, Systems, and Signal Processing, vol. 33, no. 12, pp. 3809-3837, 2014.

[26] X.-J. Xie and N. Duan, "Output tracking of high-order stochastic nonlinear systems with application to benchmark mechanical system," IEEE Transactions on Automatic Control, vol. 55, no. 5, pp. 1197-1202, 2010.

[27] X.-J. Xie, N. Duan, and C.-R. Zhao, "A combined homogeneous domination and sign function approach to output-feedback stabilization of stochastic high-order nonlinear systems," IEEE Transactions on Automatic Control, vol. 59, no. 5, pp. 1303-1309, 2014.

[28] L. Liu and X.-J. Xie, "Output-feedback stabilization for stochastic high-order nonlinear systems with time-varying delay," Automatica, vol. 47, no. 12, pp. 2772-2779, 2011.

[29] X.-J. Xie and L. Liu, "Further results on output feedback stabilization for stochastic high-order nonlinear systems with time-varying delay," Automatica, vol. 48, no. 10, pp. 2577-2586, 2012.

[30] X.-J. Xie and L. Liu, "A homogeneous domination approach to state feedback of stochastic high-order nonlinear systems with time-varying delay," IEEE Transactions on Automatic Control, vol. 58, no. 2, pp. 494-499, 2013.

[31] C. J. Qian, "A homogeneous domination approach for global output feedback stabilization of a class of nonlinear systems," in Proceedings of the American Control Conference, pp. 4708-4715, June 2005.

[32] X. J. Xie, C. R. Zhao, and N. Duan, "Further results on state feedback stabilization of stochastic high-order nonlinear systems," Science China Information Sciences, vol. 57, no. 7, pp. 1-14, 2014.

[33] F. Z. Gao, Z. Yuan, and F. S. Yuan, "State-feedback stabilization for stochastic high-order nonlinear systems with time-varying delays," Abstract and Applied Analysis, vol. 2014, Article ID 673869, 11 pages, 2014.

[34] W. T. Zha, J. Y. Zhai, and S. M. Fei, “Output feedback control for a class of stochastic high-order nonlinear systems with timevarying delays," International Journal of Robust and Nonlinear Control, vol. 24, no. 16, pp. 2243-2260, 2013.

[35] R. D. Nussbaum, "Some remarks on a conjecture in parameter adaptive control," Systems and Control Letters, vol. 3, no. 5, pp. 243-246, 1983.

[36] Y.-C. Wang, H.-G. Zhang, and Y.-Z. Wang, "Fuzzy adaptive control of stochastic nonlinear systems with unknown virtual control gain function," Acta Automatica Sinica, vol. 32, no. 2, pp. 170-178, 2006. 
[37] T. S. Li, Z. F. Li, D. Wang, and C. L. P. Chen, "Outputfeedback adaptive neural control for stochastic nonlinear time varying delay systems with unknown control directions," IEEE Transactions on Neural Networks and Learning Systems, vol. 26, no. 6, pp. 1188-1201, 2014.

[38] T. Wang, S. C. Tong, and Y. M. Li, "Robust adaptive fuzzy output feedback control for stochastic nonlinear systems with unknown control direction," Neurocomputing, vol. 106, pp. 3141, 2013. 


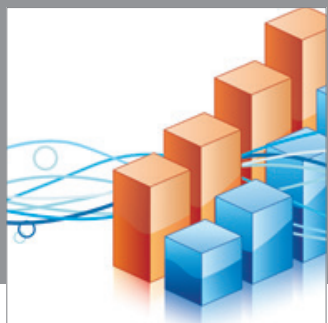

Advances in

Operations Research

mansans

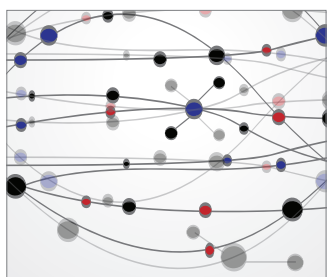

The Scientific World Journal
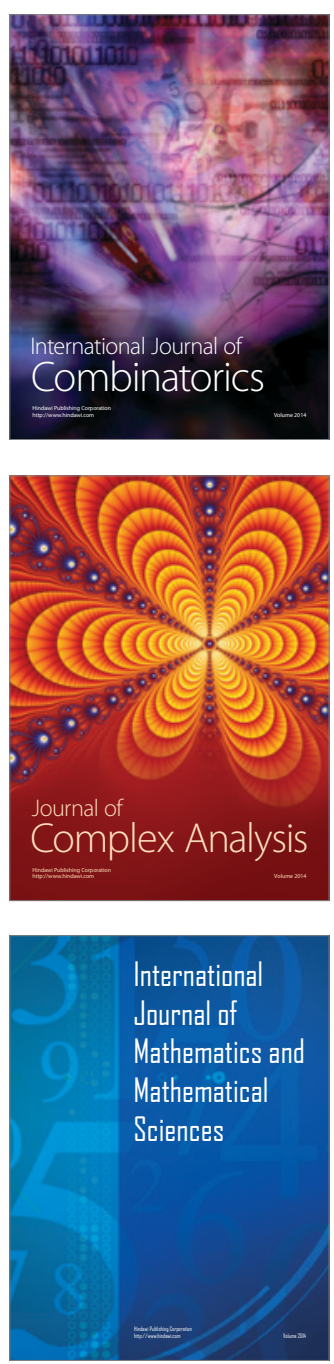
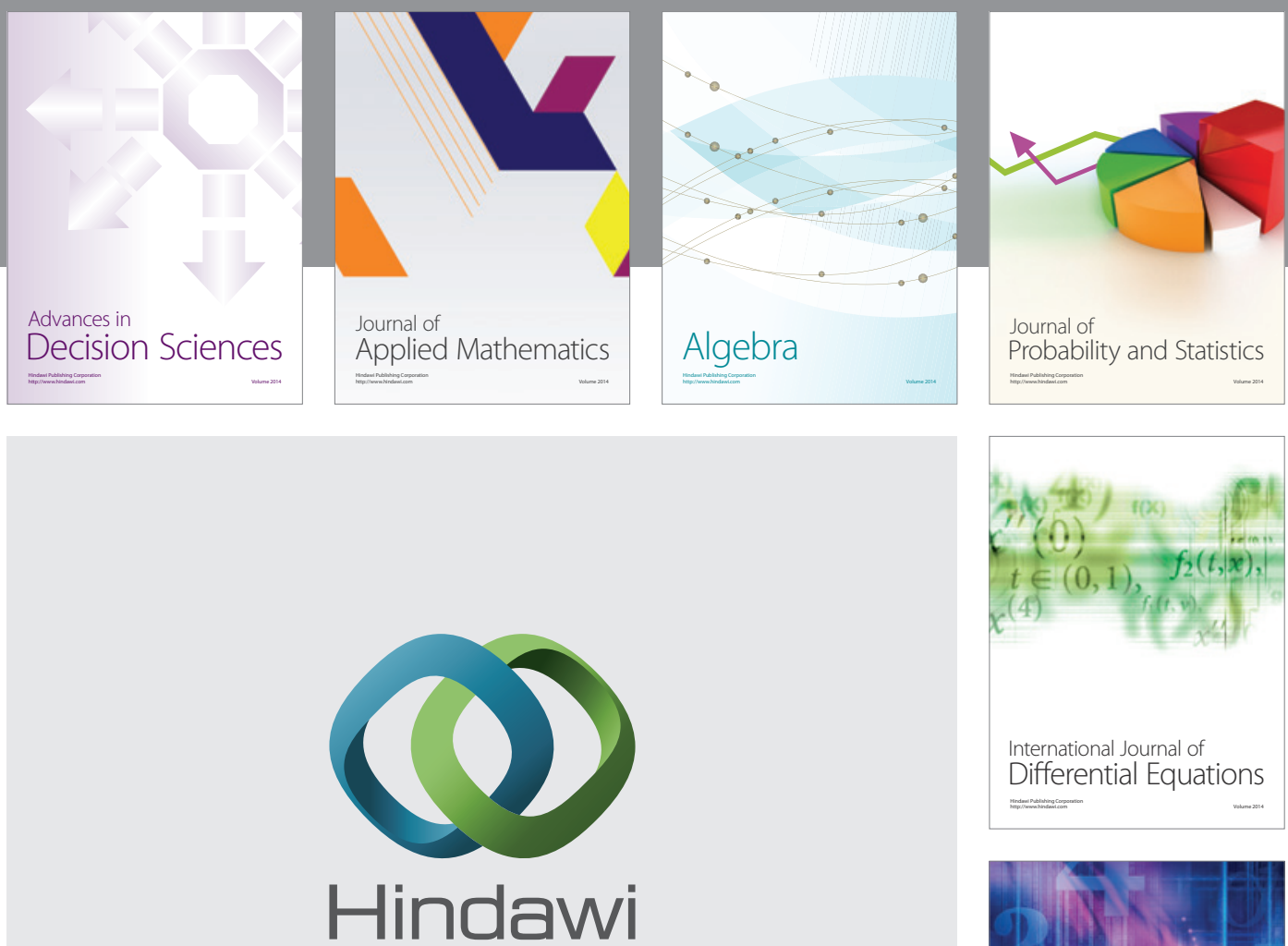

Submit your manuscripts at http://www.hindawi.com
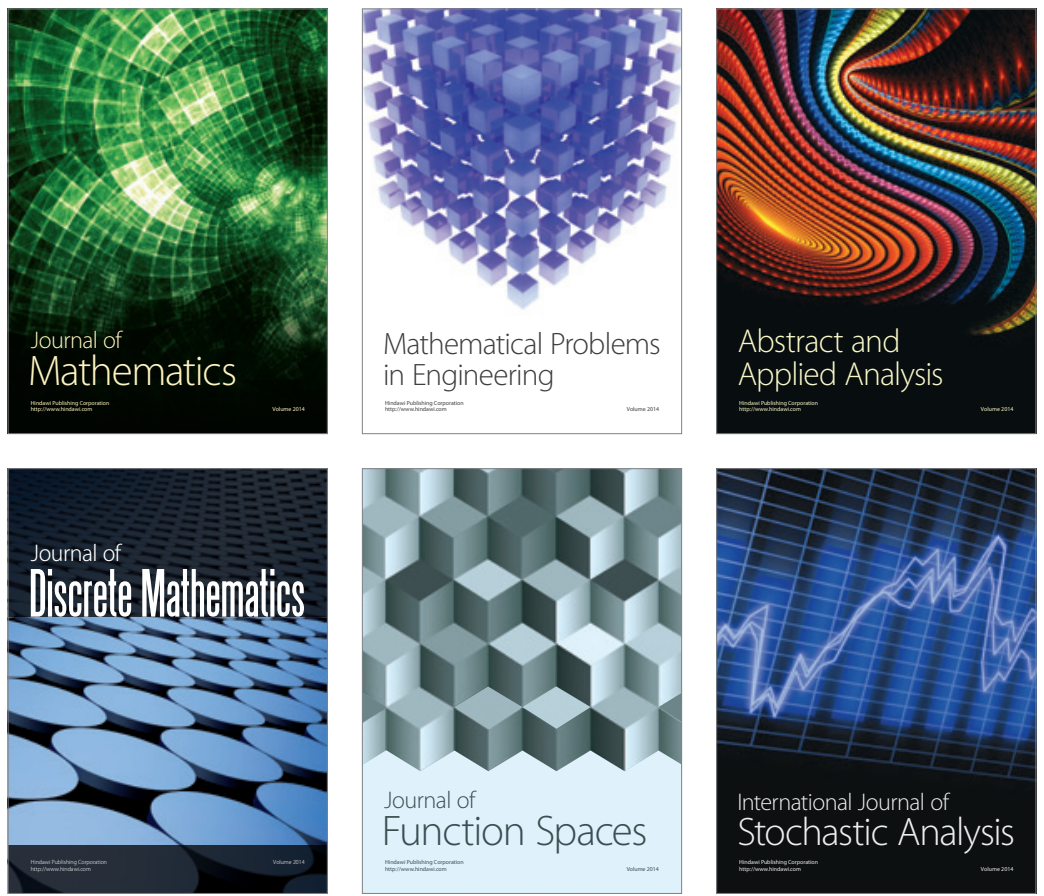

Journal of

Function Spaces

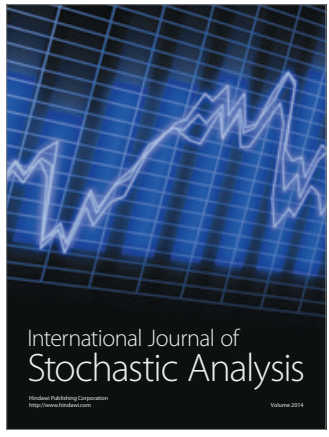

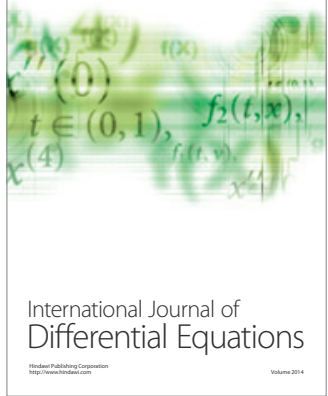
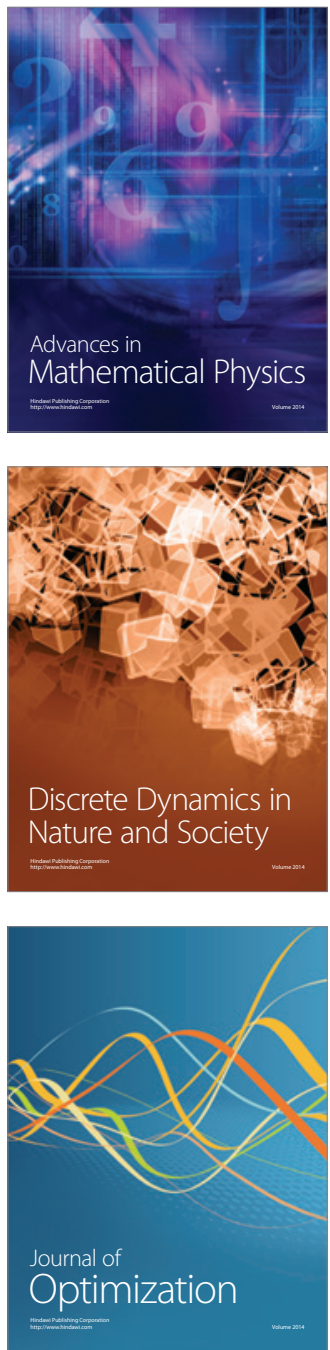